\title{
Secure Computation with Partial Message Loss ${ }^{\star}$
}

\author{
Chiu-Yuen Koo \\ Dept. of Computer Science, University of Maryland, College Park, USA \\ cykoo@cs.umd.edu
}

\begin{abstract}
Existing communication models for multiparty computation (MPC) either assume that all messages are delivered eventually or any message can be lost. Under the former assumption, MPC protocols guaranteeing output delivery are known. However, this assumption may not hold in some network settings like the Internet where messages can be lost due to denial of service attack or heavy network congestion. On the other hand, the latter assumption may be too conservative. Known MPC protocols developed under this assumption have an undesirable feature: output delivery is not guaranteed even only one party suffers message loss.

In this work, we propose a communication model which makes an intermediate assumption on message delivery. In our model, there is a common global clock and three types of parties: (i) Corrupted parties (ii) Honest parties with connection problems (where message delivery is never guaranteed) (iii) Honest parties that can normally communicate but may lose a small fraction of messages at each round due to transient network problems. We define secure MPC under this model. Output delivery is guaranteed to type (ii) parties that do not abort and type (iii) parties.

Let $n$ be the total number of parties, $e_{f}$ and $e_{c}$ be upper bounds on the number of corrupted parties and type (ii) parties respectively. We construct a secure MPC protocol for $n>4 e_{f}+3 e_{c}$. Protocols for broadcast and verifiable secret sharing are constructed along the way.
\end{abstract}

\section{Introduction}

The study of secure multiparty computation (MPC) was initiated by Yao[26] in the 2-party setting and extended to the multiparty setting by Goldreich, Micali and Wigderson[16]. Roughly speaking, a set of $n$ parties wants to jointly compute a function $g$ of their (private) inputs. However, up to $t$ of them are corrupted by an adversary. The requirements are that (i) non-corrupted parties obtain their outputs and (ii) the adversary learns nothing but the outputs of the corrupted parties.

Several communication models are considered in the current body of work, giving rise to different feasibility results, as follows.

1. Common global clock and message delivery within bounded time: This is the synchronous model. Protocol execution consists of rounds. It is assumed that the duration of one round is sufficient for a message to be sent and delivered from one party to another.

^ Supported by NSF Trusted Computing grant \#0310751. 
If $t<n / 3$, then information-theoretically secure MPC protocols exist $[3,11]$ in a point-to-point network. If we assume the existence of a broadcast channel, then information-theoretically secure MPC protocols exist for $t<$ $n / 2[22,12,1]$.

If the definition of secure MPC is relaxed such that non-corrupted parties are not guaranteed to receive their outputs (i.e., without guarantee on output delivery), then computationally secure MPC protocols exist $[16,17]$ for any $t<n$.

2. Eventual message delivery without bound on delivery time: This is the asynchronous model with eventual message delivery assumption. There is no assumed bound on the network latency. Under this communication model, information-theoretically secure MPC protocols exist for $t<n / 3[2,4,7]{ }^{1}$

3. No eventual message delivery: This is known as the "message blocking" model and is the communication model considered in $[8,10]$. There is no assumed bound on the network latency and any message can be lost. Assuming a common reference string, an Universally Composable[8] secure multiparty computation protocol (without guarantee on output delivery) exists for any $t<n$ in the computational setting[10].

4. Local clock with bounded drift and message delivery within bounded time: This is the timing model considered in [18]. It is assumed that the local clocks of the parties proceed at the same rate and an upper bound is known on the network latency. Under this model, an universally composable secure multiparty computation protocol (without guarantee on output delivery) exists for any $t<n$ in the computational setting[18]. It is worth mentioning that the security of the protocol holds as long as the assumption about local clocks holds. The network latency assumption is used to ensure non-triviality of the protocol.

We note that the results mentioned in models 1-3 hold for an adaptive adversary while the result mentioned in model 4 holds for a static adversary.

\subsection{Applicability of Existing Models to General Network Setting}

We discuss whether the assumptions made in the existing models are applicable to general network settings like the internet.

- Message delivery within bounded time: In a real network setting, an upper bound on the network latency can be very large. Even worse, as noted in [18], any reasonable bound is unlikely to hold, and hence the security of a protocol can be compromised. Consider the following scenario: $n$ parties execute the protocol by Ben-Or, Goldwasser and Wigderson[3] and the adversary corrupts $n / 6+1$ parties. In the first round, parties share their private inputs using a $(n / 3+1)$-out- $n$ secret sharing scheme. Suppose an uncorrupted party

\footnotetext{
${ }^{1}$ We note that this result cannot be translated to the synchronous model since the definition of secure asynchronous computation is different from the synchronous counterpart.
} 
$p_{i}$ suffers network congestion: $n / 6$ uncorrupted parties fail to receive their shares of $p_{i}$ 's input in time. These $n / 6$ uncorrupted parties will broadcast complaints in the next round and $p_{i}$ will reveal the corresponding shares in the round after the next round. ${ }^{2}$ The adversary will then have enough shares to reconstruct the private input of $p_{i}$.

- Eventual message delivery: This is a weaker assumption than the previous one. Under this assumption, we can have secure MPC protocols that guarantee output delivery. However, given the current form of the internet, this assumption may still be too strong. Messages sent to a party can be lost due to denial of service(DoS) attack[19] or heavy network congestion.

- Messages can be blocked: Under the message blocking model, known MPC protocols have an undesirable feature: output delivery cannot be guaranteed when one party suffers message loss (even if all parties are honest). An adversary can then have a simple strategy to prevent parties from receiving the outputs: carrying out a denial of service attack on a chosen party.

The assumption that every message can be lost may be conservative. Depending on the scenarios, it may be reasonable to assume a few, but not many, parties suffer from from DoS attack or network congestion at the same time.

In this work, we propose a communication model that is an intermediate between eventual message delivery model and message blocking model. Under this model, we construct a secure multiparty computation protocol that guarantees output delivery to all parties except those experience severe message loss.

\subsection{Our Model}

Three assumptions are made in our model:

1. A common global clock: Given the current state of art for modern network, we believe it is reasonable to assume the existence of a common global clock $^{3}$. Protocol execution consists of rounds. Every party knows when a round starts and ends.

2. Three type of parties: We assume that there are three types of parties in the network:

- Corrupted parties who are controlled by an adversary.

- Honest parties with connection problems (where message delivery is never guaranteed). An honest party that fails to contact the common global clock belongs to this category.

- Honest parties that can normally communicate but at each round they may fail to send/receive a small fraction of messages due to transient network problems.

\footnotetext{
${ }^{2}$ We remark that this is a simplification of what actually happens.

${ }^{3}$ For instance, NIST has provided such a service: http://tf.nist.gov/timefreq/ service/its.htm?
} 
From now on, we will address the second type of parties as constrained parties and the third type of parties as fault-free parties. A constrained party does not necessarily realize that it suffers from connection problem.

3. A time bound related to network latency: We assume that there is a time bound $\Delta$ such that

- Duration of a round is equal to $\Delta$.

- Any fault-free party $p$ can successfully communicate with all but $\delta$ fraction of fault-free parties in any round (i.e., message transmission from it to another party takes time less than $\Delta$ and vice versa). The set of fault-free parties $p$ can communicate with may vary each round.

\subsection{Discussions of Our Model and Related Works}

The first assumption is also made in the synchronous model.

The second assumption is inspired by the previous work in distributed computing. Thambidurai and Park[24], and independently Garay and Perry[14], introduced the concept of hybrid failure model which allows a mix of different degrees of failures. Our second assumption can be viewed as assuming a mix of omission $[21,20]$ and Byzantine failures, which is a more general assumption than the previous ones considered in the literature.

In $[23,25,6]$, protocols for broadcast and consensus are considered in a communication model where the edges can be faulty (in addition to faulty nodes). In [9], Canetti, Halevi and Herzberg considered the problem of maintaining authenticated communication over untrusted communication channels, where an adversary can corrupt links for transient periods of time. In both lines of work, there is a bound on the number of faulty links connected to an honest party. On the other hand, our model captures the scenario when an honest party suffers from arbitrary message loss.

We believe the third assumption is more realizable than assuming a time bound on the maximum latency, and yet it is sufficient to guarantee output delivery of fault-free parties.

If constrained parties are absent, then our model is reduced to the standard synchronous model. On the other hand, if fault-free parties are absent, then our model can be viewed as a message blocking model with time-out.

\subsection{Our Results}

We define secure multiparty computation under our communication model. We defer the formal definition to the next section, but roughly speaking, we require the followings: (i) the fault-free parties always receive their outputs; (ii) if a constrained party does not realize that it suffers from connection problems, then it will receive its output, otherwise, it aborts; (iii) the adversary learns nothing but the outputs of the corrupted parties.

We consider an adaptive, rushing adversary. The adversary is adaptive in the sense that in any round, it can turn a fault-free party into a constrained party or into a corrupted party. In each round, the adversary has the power to decide 
the set of messages a constrained party can receive/send and the set of parties a fault-free party can communicate with (subject to the $\delta$ constraint). We assume each pair of parties is connected by a secure channel.

Let $e_{f}$ be an upper bound on the number of corrupted parties; $e_{c}$ be an upper bound on the number of constrained parties; $n$ be the total number of parties. For $\delta<\frac{1}{6}$, we construct an information-theoretically secure MPC protocol for $n>4 e_{f}+3 e_{c}$. We define broadcast and verifiable secret sharing (VSS) under the new communication model along the way. The results are as follows (all results hold for $\left.\delta<\frac{1}{6}\right)$ :

- A broadcast protocol for $n>3 e_{f}+2 e_{c}$; we also have a different broadcast protocol for $n \geq 3 e_{f}+2 e_{c}$ if it is known that $e_{f}, e_{c} \geq 1$.

- A VSS protocol for $n>4 e_{f}+3 e_{c}$.

\section{Notations, Definitions and Overview}

\section{$2.1 \quad$ Notations}

We use two special symbols $\phi$ and $\perp$ in the paper. $\phi$ is a special symbol denoting the failure of receiving a valid message. During a protocol execution, if a party $p_{r}$ fails to receive a valid message from another party $p_{s}$, then we say $p_{r}$ receives $\phi$. If $p_{r}$ is not a corrupted parties and $p_{r}$ receives $\phi$ from $p_{s}$, assuming $\delta=0$, then one of the followings must hold:

1. $p_{s}$ is a corrupted party while $p_{r}$ is a fault-free party or a constrained party.

2. $p_{s}$ is a constrained party while $p_{r}$ is a fault-free party or a constrained party.

3. $p_{s}$ is a fault-free party while $p_{r}$ is a constrained party.

If $\delta>0$, then it is possible that both $p_{s}$ and $p_{r}$ are fault-free parties and yet $p_{r}$ receives $\phi$ from $p_{s}$.

$\perp$ is a special symbol denoting abortion. In any (sub-)protocol execution, if a party outputs $\perp$, then the party aborts the entire execution at that point. We also assume that a constrained party outputs $\perp$ if it fails to contact the common global clock. Our protocols are designed in such a way that only a constrained party will output $\perp$. For clarity, when we refer to an uncorrupted party $p_{i}$ in our proofs, unless otherwise specified, we implicitly assume that $p_{i}$ is a fault-free party or a constrained party who has not aborted at that point (i.e., we do not consider a constrained party that has already aborted).

\subsection{Definition of Secure Computation}

We define the secure multiparty computation using the ideal/real world paradigm. We assume the function $g$ is defined in a way such that if the input of an party is $\phi$, then the evaluation of $g$ does not depend on the input of that party and the corresponding output for that party is $\perp$. We also assume that if the output of a party is not equal to $\perp$, then its output contains the set of parties which input $\phi$. As a warm-up, we will start with the case of a non-adaptive adversary. 


\section{The Non-adaptive Case}

Ideal world: In the ideal world, there is a trusted party (TP) which carries out the evaluation of the function $g$. The evaluation consists of the following steps:

1. The adversary chooses a set of corrupted parties $\mathcal{P}^{f}$, modifies their inputs (which can become $\phi$ ) and sends them to the trusted party; the adversary chooses three sets of constrained parties $\mathcal{P}^{c_{1}}, \mathcal{P}^{c_{2}}$ and $\mathcal{P}^{c_{3}} ;^{4}$ the trusted party receives the private inputs from parties that are not in $\mathcal{P}^{f} \cup \mathcal{P}^{c_{1}}$; the trusted party receives $\phi$ as the input from the constrained parties in $\mathcal{P}^{c_{1}}$.

2. The trusted party evaluates $g$. Let $\mathcal{P}^{\phi}$ be the set of parties which send $\phi$ to the trusted party.

3. The adversary receives the outputs of the parties in $\mathcal{P}^{f}$; parties in $\mathcal{P}^{c_{1}} \cup \mathcal{P}^{c_{2}}$ receive $\perp$; other parties receive their outputs (note that the outputs contain the set $\left.\mathcal{P}^{\phi}\right)$.

Real world: In the real world, the parties execute a protocol $\Pi$ to evaluate $g$. Corrupted parties may deviate from the protocol in an arbitrary manner. Messages delivery are controlled by the adversary, subject to the constraints in our communication model.

At the end of the protocol execution, the fault-free parties and the constrained parties output their outputs from $\Pi$; the real-world adversary generates an output (which can depend on the information it gathers during the execution of $\Pi)$.

We say a protocol $\Pi$ is a secure multiparty computation protocol if the following holds: for every real world adversary $\mathcal{A}$, there exists an ideal world adversary $\mathcal{I}$ with the same set of corrupted parties and same set of constrained parties such that (1) and (2) are indistinguishable:

1. The output of $\mathcal{I}$ and the outputs of fault-free parties and constrained parties in the ideal world.

2. The output of $\mathcal{A}$ and the outputs of fault-free parties and constrained parties in the real world.

The Adaptive Case. The only difference between this case and the nonadaptive case is the definition of the ideal world. In the ideal world,

1. The adversary chooses a set of corrupted parties $\mathcal{P}^{f_{1}}$ (in an adaptive manner), modifies their inputs (which can become $\phi$ ) and sends them to the trusted party; the adversary chooses a set of constrained parties $\mathcal{P}^{c_{1}}$; the trusted party receives the private inputs from parties that are not in $\mathcal{P}^{f_{1}} \cup$ $\mathcal{P}^{c_{1}}$; the trusted party receives $\phi$ as the input from the constrained parties in $\mathcal{P}^{c_{1}}$.

2. The trusted party evaluates $g$. Let $\mathcal{P}^{\phi}$ be the set of parties who send $\phi$ to the trusted party.

${ }^{4}$ A constrained party in $\mathcal{P}^{c_{3}}$ is not distinguishable from a fault-free party in the ideal world, the set $\mathcal{P}^{c_{3}}$ is defined due to a subtle technical point. 
3. The adversary receives the outputs of the parties in $\mathcal{P}^{f_{1}}$; depending on the outputs it received, the adversary can (adaptively) choose to corrupt a new set of parties $\mathcal{P}^{f_{2}}$ and obtain their outputs; the adversary then chooses two sets of constrained parties $\mathcal{P}^{c_{2}}$ and $\mathcal{P}^{c_{3}}$; parties in $\mathcal{P}^{c_{1}}$ and $\mathcal{P}^{c_{2}}$ receive $\perp$; other uncorrupted parties receive their outputs.

At the end, the fault-free parties and the constrained parties output what they receive from the trusted party; the ideal-world adversary generates an output.

\subsection{Overview}

In section 5, we construct a MPC protocol for $n>4 e_{f}+3 e_{c}$ which uses broadcast(section 3) and VSS(section 4) as sub-protocols. We note that we assume $\delta=0$ (i.e. a fault-free party can always successfully receive messages from other fault-free parties) in sections 3,4 and 5 . In section 6 , we discuss how to extend our results to the case of $\delta<\frac{1}{6}$. In section 7 , we conclude and state some open problems.

\section{Broadcast}

\subsection{Definitions}

In broadcast, there is a distinguished sender $p_{s}$ with input $v$. We can define broadcast using the ideal/real world paradigm by specifying the ideal world as follows:

1. The adversary chooses a set of corrupted parties $\mathcal{P}^{f}$ and a set of constrained parties $\mathcal{P}^{c}$.

- If $p_{s} \in \mathcal{P}^{f}$, then the adversary obtains the value $v$ and $p_{s}$ sends a possibly modified value $v^{\prime}\left(v^{\prime}\right.$ can be equal to $\left.\phi\right)$ to the trusted party.

- If $p_{s} \in \mathcal{P}^{c}$, then the adversary sends a flag $b$ to $p_{s}$; if $b$ is equal to true, then $p_{s}$ sends $v$ to the trusted party else $p_{s}$ sends $\phi$.

- If $p_{s} \notin \mathcal{P}^{f} \cap \mathcal{P}^{c}$, then $p_{s}$ sends $v$ to the trusted party.

2. The trusted party sends the value it received from $p_{s}$ to all parties not in $\mathcal{P}^{c}$; it sends $\phi$ to all parties in $\mathcal{P}^{c}$.

However, the above definition is an overkill for our application (as a building block for VSS and MPC). If $p_{s}$ is a constrained party and it fails to broadcast the message (i.e., $p_{s}$ receives $b=$ false from the adversary and the honest parties receive $\phi$ ), then the adversary should obtain no knowledge about the message. We need some kind of secret sharing to achieve this in the real world. However, our intention is to construct a VSS protocol using broadcast, not the other way round! To solve this dilemma, we observe that in our applications of broadcast, privacy is not an issue. In more details, what we need are as follows:

- If the sender is corrupted, then all fault-free parties receive the same value $v^{\prime}\left(v^{\prime}\right.$ can be equal to $\left.\phi\right)$. A constrained party should receive $v^{\prime}$ or $\phi$. 
- If the sender is constrained, then all fault-free parties receive the same value $v^{\prime}$ ( $v^{\prime}$ has to be equal to $v$ or $\phi$ ). A constrained party should receive $v^{\prime}$ or $\phi$.

- If the sender is fault-free, then all fault-free parties receive the same value $v^{\prime}=v$. A constrained party should receive $v^{\prime}$ or $\phi$.

It will ease the designing of the VSS protocol if we place a more stringent requirement: if a constrained party does not receive $v^{\prime}$, then it aborts (i.e. outputting $\perp$ ).

More formally, we say broadcast is achieved if the followings hold:

- Agreement: If an uncorrupted party outputs $v^{\prime}(\neq \perp)^{5}$, then all fault-free parties output $v^{\prime}$.

- Correctness: If the sender is uncorrupted and an uncorrupted party outputs $v^{\prime}(\neq \perp)$, then $v^{\prime}=v$ or $v^{\prime}=\phi$. If the sender is fault-free, then all fault-free parties output $v$.

If the sender is constrained, it is possible that all fault-free parties output $\phi$. We assume a constrained sender will abort if it outputs $\phi$ in the broadcast protocol. We reduce the broadcast problem to the consensus problem. In consensus, every party $p_{i}$ has an input $v_{i}$. Consensus is achieved if the followings hold:

- Agreement: All fault-free parties output a common value $v$. A constrained party either outputs $v$ or $\perp$ (abort).

- Persistence: If all fault-free parties have the same input $v^{\prime}$, then $v=v^{\prime}$.

For the rest of the section, we focus on the case where the domain of values is restricted to $\{0,1\}$. It is easy to see that if we have a broadcast protocol for a single bit, then we can have broadcast protocol for a $\ell$-bit string by running the bit-protocol $\ell$-times sequentially. For a bit $b$, we denote its complement by $\bar{b}$.

\subsection{Reducing Broadcast to Consensus}

Under our communication model, broadcast cannot be achieved by simply having the sender sending its value to all parties and then running the consensus protocol. The problem is that the sender could be constrained and fault-free parties may not receive the value from the sender. Nevertheless, we show the following:

\section{Lemma 1. Consensus implies broadcast.}

Proof. We construct a broadcast protocol from any consensus protocol:

1. Sender $p_{s}$ sends the bit $v$ to all parties. Let $b_{i}$ be the bit $p_{i}$ received from $p_{s}$. If $p_{i}$ does not receive anything from the sender, then sets $b_{i}=0$.

2. Parties execute the consensus protocol. Each party $p_{i}$ enters the protocol with input $b_{i}$ and let $b_{i}^{*}$ be the output. If $b_{i}^{*}=\perp$, then $p_{i}$ outputs $\perp$ and aborts.

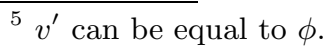


3. If $v=b_{s}^{*}$, then $p_{s}$ sends 1 to all parties; otherwise, $p_{s}$ does not send anything.

4. If $p_{i}$ receives 1 from the sender, then sets $d_{i}=1$ else sets $d_{i}=0$. Parties execute the consensus protocol again. This time, $p_{i}$ enters the protocol with input $d_{i}$ and lets $d_{i}^{*}$ be the corresponding output. If $d_{i}^{*}=1$, then $p_{i}$ outputs $b_{i}^{*}$ else if $d_{i}^{*}=0$, then $p_{i}$ outputs $\phi$ else $p_{i}$ outputs $\perp\left(d_{i}^{*}=\phi\right.$ for the last case) .

The consensus protocol is run twice in the construction. Roughly speaking, the first execution establishes a common value among the parties. However, if the sender is constrained, then the established value may be different from $v$. The second execution is to determine if the sender is "happy" with the established value. If the sender is not happy, then all parties output $\phi$. The formal proof proceeds as follows:

Agreement: (i) If an uncorrupted party outputs $b \notin\{\phi, \perp\}$, then by the agreement property of consensus, $d_{i}^{*}=1$ and $b_{i}^{*}=b$ for all fault-free parties $p_{i}$. Hence all fault-free parties output $b$. (ii) If an uncorrupted party outputs $\phi$, then $d_{i}^{*}=0$ for all fault-free parties $p_{i}$. Hence all fault-free parties output $\phi$.

Correctness: Consider two cases: (i) a fault-free sender and (ii) a constrained sender. (i) If the sender is fault-free, then all fault-free parties receive $v$ from the sender in step 1. By the persistence property of consensus, all fault-free parties have $b_{i}^{*}=v$. Hence all fault-free parties $p_{i}$ receive 1 from the sender in step 3 and sets $d_{i}=1$ in step 4 . By the persistence property of consensus again, $d_{i}^{*}=1$. Hence a fault-free party outputs $v$. (ii) If the sender $p_{s}$ is constrained, consider two sub-cases: (a) $b_{s}^{*}=v$ (b) $b_{s}^{*} \neq v$. For case (a), a fault-free party $p_{i}$ may or may not receive 1 from $p_{s}$ in step 3 and $d_{i}^{*}$ may equal to 0 or 1 . However, note that $b_{i}^{*}=b_{s}^{*}=v$. Therefore, $p_{i}$ either outputs $v$ or $\phi$. For case (b), $p_{s}$ does not send anything in step 3 . Hence all fault-free parties $p_{i}$ enter the consensus protocol in step 4 with input $d_{i}=0$. By the persistence property of consensus, $d_{i}^{*}=0$. Therefore all uncorrupted parties output $\phi$.

\subsection{Consensus for $n>3 e_{f}+2 e_{c}$}

Following the principle of Berman et al.[5], the construction of the consensus protocol is done through constructing protocols for weaker consensus variants: weak consensus, graded consensus, king consensus and then consensus. In all these (sub-)protocols, we only need to know the number of fault-free parties $e_{f f} \stackrel{\text { def }}{=} n-e_{f}-e_{c}$, but not $e_{f}$ and $e_{c}$; moreover, we only require authenticate (but not secure) point-to-point channels. For all these (sub-)protocols, $p_{i}$ has an input bit and we denote it as $b_{i}$.

Weak Consensus. We say weak consensus is achieved if the following two conditions hold:

- Persistence: If all fault-free parties have the same input bit $b$, then all faultfree parties output $b$.

- Agreement: If an uncorrupted party outputs $b \in\{0,1\}$, then all uncorrupted parties output $b$ or 2 . 
1. $p_{i}$ sends $b_{i}$ to all parties.

2. Let $X_{i}^{0}$ and $X_{i}^{1}$ be the number of 0 and 1 received by $p_{i}$ respectively. If $X_{i}^{0} \geq e_{f f}, p_{i}$ outputs 0 , else if $X_{i}^{1} \geq e_{f f}, p_{i}$ outputs 1 else $p_{i}$ outputs 2 .

Lemma 2. Protocol WConsensus achieves weak consensus for $n>3 e_{f}+2 e_{c}$.

Proof. Persistence: Note that $e_{f f}>\frac{n}{2}$. If all fault-free parties $p_{i}$ have the same input bit $b$, then $X_{i}^{b} \geq e_{f f}$ and $X_{i}^{\bar{b}}<e_{f f}$. Hence $p_{i}$ will output $b$. Agreement: Suppose there exists two uncorrupted parties $p_{i}$ and $p_{j}$ outputting 0 and 1 respectively. Then $\left|X_{i}^{0} \cap X_{j}^{1}\right| \geq e_{f f}-\left(e_{f}+e_{c}\right)=n-e_{f}-e_{c}-\left(e_{f}+e_{c}\right)>e_{f}$. Therefore, there exists more than $e_{f}$ parties sending different bits to $p_{i}$ and $p_{j}$ in round 1 . This is a contradiction since there are at most $e_{f}$ corrupted parties.

Graded consensus. In graded consensus, every party $p_{i}$ outputs a bit along with a grade $g_{i}$. Graded consensus is achieved if the following three conditions are satisfied:

- Persistence: If all fault-free parties have the same input bit $b$, then all faultfree parties output $b$ with $g=1$.

- Agreement: If an uncorrupted party outputs $b$ with $g=1$, then all fault-free parties output $b$, all constrained parties output $b$ or $\perp$.

- Completeness: No fault-free party outputs $\perp$.

$\underline{\text { Protocol GConsensus }\left(p_{i}, b_{i}, e_{f f}\right)}$

1. $p_{i}$ sends the output of WConsensus $\left(p_{i}, b_{i}, e_{f f}\right)$ to all parties.

2. Let $X_{i}^{0}, X_{i}^{1}$ and $X_{i}^{2}$ be the number of 0,1 and 2 received by $p_{i}$ respectively. If $\max \left\{X_{i}^{0}, X_{i}^{1}\right\}+X_{i}^{2}<e_{f f}$, then $p_{i}$ outputs $\perp$ and abort.

If $X_{i}^{0} \geq e_{f f}, p_{i}$ outputs 0 with $g_{i}=1$, else if $X_{i}^{1} \geq e_{f f}, p_{i}$ outputs 1 with $g_{i}=1$, else if $X_{i}^{0}>X_{i}^{1}, p_{i}$ outputs 1 with $g_{i}=0$, else $p_{i}$ outputs 0 with $g_{i}=0$.

Lemma 3. Protocol GConsensus achieves graded consensus for $n>3 e_{f}+2 e_{c}$.

Proof. Persistence: If all fault-free parties have the same input bit $b$, then following the persistence property of weak consensus, they output the same bit $b$ in WConsensus. For a fault-free party $p_{i}, X_{i}^{b} \geq e_{f f}$ and $X_{i}^{b}<e_{f f}$. Therefore $p_{i}$ outputs $b$ with $g_{i}=1$. Agreement: If an uncorrupted party $p_{i}$ outputs $b$ with $g_{i}=1$, then $X_{i}^{b} \geq e_{f f}$. Hence at least $e_{f f}-e_{f}$ uncorrupted parties have $b$ as the output of WConsensus. Following the agreement property of weak consensus, the number of uncorrupted parties that output $\bar{b}$ in WConsensus is equal to 0 . By counting, the number of uncorrupted parties that output 2 in WConsensus is at most $e_{f f}+e_{c}-\left(e_{f f}-e_{f}\right)=e_{c}+e_{f}$. Assume on contrary that there exists an uncorrupted party $p_{j}$ outputs $\bar{b}$ in GConsensus. Then $X_{j}^{\bar{b}} \geq X_{j}^{b}$. Note that all $\bar{b} p_{j}$ received in step 1 are from corrupted parties. Therefore, $X_{j}^{2} \leq e_{f}+e_{c}+\left(e_{f}-X_{j}^{\bar{b}}\right)$. 
( $e_{f}+e_{c}$ corresponds to the number of 2 received due to uncorrupted parties; $e_{f}-X_{j}^{\bar{b}}$ corresponds to the number of 2 received due to corrupted parties.) But $X_{j}^{\bar{b}}+X_{j}^{2} \leq X_{j}^{\bar{b}}+e_{f}+e_{c}+\left(e_{f}-X_{j}^{\bar{b}}\right)=2 e_{f}+e_{c}<e_{f f}$ as $n>3 e_{f}+2 e_{c}$ and $e_{f f}=n-e_{f}-e_{c}$. Therefore, $\max \left\{X_{j}^{0}, X_{j}^{1}\right\}+X_{j}^{2}<e_{f f}, p_{j}$ should output $\perp$ instead. Contradiction. Completeness: By the agreement property of weak consensus, for some bit $b$, each fault-free party has either $b$ or 2 as the output of WConsensus. Therefore, for a fault-free party $p_{i}, \max \left\{X_{i}^{0}, X_{i}^{1}\right\}+X_{i}^{2} \geq e_{f f}$. Hence no fault-free party outputs $\perp$.

King Consensus. In king consensus, there is a designated party $p_{k}$ known as the king. King consensus is achieved if the followings hold:

- Persistence: If all fault-free parties have the same input bit $b$, then all uncorrupted parties that do not abort output $b$.

- Correctness: If the king $p_{k}$ is fault-free, then all uncorrupted parties that do not abort output the same bit.

- Completeness: No fault-free party outputs $\perp$.

$\underline{\text { Protocol KConsensus }_{p_{k}}\left(p_{i}, b_{i}, e_{f f}\right)}$

1. Let $\left(v_{i}, g_{i}\right)$ be the output of GConsensus $\left(p_{i}, b_{i}, e_{f f}\right)$. If $\left(v_{i}, g_{i}\right)=\perp$, then $p_{i}$ outputs $\perp$.

2. $p_{k}$ sends $v_{k}$ to all parties.

3. If $\left(g_{i} \neq 1\right)$ and $p_{i}$ receives $v_{k}$ from $p_{k}$ and $v_{k} \neq \phi$, then $p_{i}$ sets $v_{i}=v_{k}$.

4. Let $\left(v_{i}^{\prime}, g_{i}^{\prime}\right)$ be the output of GConsensus $\left(p_{i}, v_{i}, e_{f f}\right) \cdot p_{i}$ outputs $v_{i}^{\prime}$.

Lemma 4. Protocol KConsensus achieves king consensus for $n>3 e_{f}+2 e_{c}$.

Proof. Persistence: If all fault-free parties have the same input bit $b$, then by the persistence property of graded consensus, all fault-free parties $p_{i}$ have $(b, 1)$ as the output of the first execution of GConsensus, i.e., $v_{i}=b$ and $g_{i}=1$. Since $g_{i}=1, v_{i}$ will not be modified in step 3. All fault-free parties enter the second execution of GConsensus with the same input $b$. By the persistence and the agreement properties of graded consensus, all uncorrupted parties output the bit $b$ in KConsensus. Correctness: Suppose $p_{k}$ is a fault-free party. Consider two cases: (i) there exists a fault-free party $p_{i}$ with $g_{i}=1$ by the end of step 1 . (ii) all fault-free parties $p_{i}$ have $g_{i}=0$ by the end of step 1. For case (i), following the agreement property of graded consensus, all fault-free parties $p_{j}$ (including $p_{k}$ ) have the same value for $v_{j}$ (i.e., $v_{j}=v_{i}=v_{k}$ ) by the end of step 1 . It does not matter whether $p_{j}$ resets its value in step 3 . For case (ii), all fault-free parties $p_{j}$ receive $v_{k}$ from $p_{k}$ in step 3 and set $v_{j}=v_{k}$. Combining two cases, all fault-free parties enter the second execution of GConsensus with the same input $v_{k}$. Following the persistence and agreement properties of graded consensus, all uncorrupted parties output $v_{k}$ in KConsensus. Completeness: Completeness of KConsensus follows the completeness of graded consensus since no fault-free party will output $\perp$ in the executions of GConsensus. 
Consensus. We show how to construct a consensus protocol from a king consensus protocol:

$\underline{\text { Procotol Consensus }\left(p_{i}, b_{i}, e_{f f}\right)}$

1. Set $b_{i}^{\prime}=b_{i}$.

2. for $k=1$ to $n-e_{f f}+1$ do:

(a) Set $b_{i}^{\prime}$ to the output of KConsensus $p_{p_{k}}\left(p_{i}, b_{i}^{\prime}, e_{f f}\right)$.

(b) If $b_{i}^{\prime}=\perp$, then $p_{i}$ outputs $\perp$ and abort.

3. $p_{i}$ outputs $b_{i}^{\prime}$.

Theorem 1. Protocol Consensus achieves consensus for $n>3 e_{f}+2 e_{c}$.

Proof. Persistence: If all fault-free parties enter the protocol Consenus with the same input bit $b$, then by the persistence property of king consensus, all uncorrupted parties that do not abort output the same bit $b$. Agreement: Note that $n-e_{f f}+1=e_{f}+e_{c}+1$. There exists a fault-free party $p_{i} \in\left\{p_{1}, \ldots, p_{e_{f}+e_{c}+1}\right\}$. By the correctness property of king consensus, all fault-free parties will have the same value for $b^{\prime}$ after KConsensus $p_{i}$ is run. Agreement then follows from the persistence property of king consensus.

\subsection{Consensus for $n \geq 3 e_{f}+2 e_{c}, e_{f}, e_{c} \geq 1$}

If the values of $e_{f}$ and $e_{c}$ are known a priori, then we can improve the bound in Theorem 1. On a high level, the construction takes two steps. First, based on the consensus protocol we have for $n>3 e_{f}+2 e_{c}$, we construct a weak broadcast (to be defined) protocol for $n \geq 3 e_{f}+2 e_{c}$. Second, we convert a weak broadcast protocol into a consensus protocol.

Weak Broadcast. In weak broadcast, there is a sender $p_{s}$ with an input bit $b$. Weak broadcast is achieved if the following two conditions hold:

- Agreement: All fault-free parties output a common bit $b^{\prime}$.

- Correctness: If the sender is fault-free, then $b=b^{\prime}$.

Note that in weak broadcast, we do not concern the outputs of constrained parties. Due to lack of the space, we omit the description of the protocol. The details will appear in the full version ${ }^{6}$.

From weak broadcast to consensus. Once we have a protocol for weak broadcast, it is easy to construct a consensus protocol:

1. Each party $p_{i}$ weak-broadcasts the input bit $b_{i}$ using the protocol WBroadcast.

2. If the majority of the broadcasted bits is 1 , then $p_{i}$ sets $b_{i}^{\prime}=1$ else $p_{i}$ sets $b_{i}^{\prime}=0 . p_{i}$ sends $b_{i}^{\prime}$ to all parties.

${ }^{6}$ A preliminary full version is available at the author's homepage: http://www. cs.umd.edu/ cykoo 
3. Let $X_{i}^{0}$ and $X_{i}^{1}$ be the number of 0 and 1 received by $p_{i}$ in last round respectively. If $X_{i}^{0}>\frac{1}{2} n$, then $p_{i}$ outputs 0 else if $X_{i}^{1}>\frac{1}{2} n$, then $p_{i}$ outputs 1 else $p_{i}$ outputs $\perp$.

Since all fault-free parties $p_{i}$ have the same output in weak broadcasts, they will have the same value for $b_{i}^{\prime}$. In particular, if all of them have the same input bit $b$, then $b_{i}^{\prime}=b$. As the majority of parties are fault-free, if an uncorrupted party $p_{j}$ receives $>\frac{1}{2}$ copies of $b_{j}^{\prime} \in\{0,1\}$ in round 2 , then $b_{j}^{\prime}=b_{i}^{\prime}$ for any fault-free party $p_{i}$. Therefore both persistence and agreement properties hold. Hence we have the following:

Theorem 2. There is a consensus protocol for $n \geq 3 e_{f}+2 e_{c}, e_{f} \geq 1, e_{c} \geq 1$, assuming the values of $e_{f}$ and $e_{c}$ are known a priori.

\section{Verifiable Secret Sharing (VSS)}

In verifiable secret sharing (VSS), there is a special party $p_{d}$ known as the dealer. The dealer holds a secret $s$. A VSS protocol consists of two phases: a sharing phase and a reconstruction phase. In the sharing phase, the dealer shares the secret with other parties. Parties may disqualify a non fault-free dealer. If the dealer is not disqualified, then in the reconstruction phase, the parties reconstruct a value based on their views in the sharing phase.

In our case, VSS protocol is used as a tool for multiparty computation. Our definition requires a VSS protocol to have the verifiable secret and polynomial sharing property[15]. In this section, we assume the values of $e_{f}$ and $e_{c}$ are known a priori. We say a protocol achieves verifiable secret sharing if the followings hold:

- Privacy: If the dealer is uncorrupted, then the view of the adversary during the sharing phase reveals no information on $s$.

- Agreement: If an uncorrupted party disqualifies the dealer, then all uncorrupted parties that do not abort disqualify the dealer.

- Commitment: If the dealer is not disqualified, then there exists a polynomial $h^{\prime}(x)$ of degree $e_{f}$ such that at the end of the sharing phase, all fault-free parties $p_{i}$ (locally) output $h^{\prime}(i)$; a constrained party $p_{j}$ which does not abort outputs $h^{\prime}(j)$. All uncorrupted parties that do not abort output $h^{\prime}(0)$ in the reconstruction phase.

- Correctness: No fault-free party will abort the protocol. A fault-free dealer will not be disqualified while a constrained dealer may be disqualified. But if an uncorrupted dealer is not disqualified, then $h^{\prime}(0)=s$.

Theorem 3. Assuming the values $e_{f}$ and $e_{c}$ are known a priori, there exists a VSS protocol for $n \geq 4 e_{f}+3 e_{c}+1$.

Proof. We construct a VSS protocol with the above resilience. The protocol is based on the bivariate solution of Feldman-Micali[13]. We start by giving a high level description of the protocol. In round 1, the dealer shares the secret via a random bivariate polynomial of degree $e_{f}+1$. If the dealer is constrained, then 
a fault-free party may not receive its entitled share. However, unlike [13], the fault-free party cannot take a default value since it will not be on the polynomial and correctness will be violated. Instead, a party broadcasts "receive" in round 2 if it has received its entitled share. Let $\mathcal{G}$ be the group of parties who broadcast "receive". If $|\mathcal{G}|$ is too small, then the dealer is disqualified. Otherwise, the parties within $\mathcal{G}$ proceed to verify if the dealer has shared a valid secret, using a similar approach as in [13](with suitable modifications to tolerate the presence of constrained parties). The parties that are not in $\mathcal{G}$ will not take part in the verification. After the verification, if the dealer is not disqualified, then all parties in $\mathcal{G}$ have shares correspond to a valid secret. The parties outside $\mathcal{G}$ then compute their shares by interpolating the shares from the parties in $\mathcal{G}$ (here we exploit the fact that the secret is shared using a bivariate polynomial).

We assume the secret $s$ is taken from some finite field $\mathcal{F}$. In the following, if the dealer broadcasts a value and the parties receive $\phi$ as the output, then we implicitly assume that all parties disqualify the dealer.

$\underline{\operatorname{VSS}-s h a r e\left(p_{d}\right)}$

1. The dealer chooses a random bivariate polynomial $f$ of degree at most $e_{f}$ in each variable such that $f(0,0)=s$. The dealer sends to party $p_{i}$ the polynomials $g_{i}(x) \stackrel{\text { def }}{=} f(x, i)$ and $h_{i}(x) \stackrel{\text { def }}{=} f(i, x)$.

2. $p_{i}$ broadcasts " 0 " if it does not receive $g_{i}(x)$ and $h_{i}(x)$ from the dealer (or $g_{i}(x)$ and $h_{i}(x)$ are not polynomials of degree $\left.e_{f}\right)$, otherwise $p_{i}$ broadcasts "1". Let $\mathcal{G}$ be the group of parties which broadcast "1". If $|\mathcal{G}|<3 e_{f}+2 e_{c}+$ 1 , then the dealer is disqualified. Otherwise, each party $p_{i} \in \mathcal{G}$ does the following:

(a) For every party $p_{j} \in \mathcal{G}, p_{i}$ sends $g_{i}(j)$ and $h_{i}(j)$ to $p_{j}$. Let $g_{j, i}^{\prime}$ and $h_{j, i}^{\prime}$ be the two values received by $p_{i}$ from $p_{j} \in \mathcal{G} . p_{i}$ aborts if it receives values from less than $2 e_{f}+e_{c}+1$ parties.

(b) For every party $p_{j} \in \mathcal{G}$, if $\left(g_{j, i}^{\prime} \neq h_{i}(j)\right.$ and $\left.g_{j, i}^{\prime} \neq \phi\right)$ or $\left(h_{j, i}^{\prime} \neq g_{i}(j)\right.$ and $\left.h_{j, i}^{\prime} \neq \phi\right)$, then $p_{i}$ broadcasts "complaint : i,j" else $p_{i}$ broadcasts "no complaint: $\mathrm{i}, \mathrm{j}$ ". Note that if an uncorrupted $p_{i}$ broadcasts "complaint: $\mathrm{i}, \mathrm{j}$ ", then $p_{j}$ or the dealer is corrupted.

(c) The dealer broadcasts $f_{j, k} \stackrel{\text { def }}{=} f(j, k)$ and $f_{k, j} \stackrel{\text { def }}{=} f(k, j)$ if "complaint: $\mathrm{j}, \mathrm{k} "$ is broadcasted by a party $p_{j} \in \mathcal{G}$.

(d) If there exists a $j$ such that (i) $f_{j, i}$ and $f_{i, j}$ are revealed in last step and (ii) $f_{j, i} \neq g_{i}(j)$ or $f_{i, j} \neq h_{i}(j)$, then $p_{i}$ broadcasts "complaint", otherwise $p_{i}$ broadcasts "okay". (If an uncorrupted $p_{i}$ broadcasts "complaint", then the dealer must be corrupted. On the other hand, if the dealer is uncorrupted and $p_{i}$ broadcasts "complaint", then $p_{i}$ must be corrupted.)

(e) If $p_{i}$ broadcasts "complaint" in last step, then the dealer broadcasts $g_{i}(x)$ and $h_{i}(x)$.

(f) $p_{i}$ broadcasts "reject" if one of the followings hold:

$-p_{i}$ broadcasts "complaint" in step $2(\mathrm{~d})$

- There exists a public polynomial $g_{k}(x)$ and $h_{k}(x)$ such that $g_{k}(i) \neq$ $h_{i}(k)$ or $h_{k}(i) \neq g_{i}(k)$ 
- The dealer does not respond to the complaints broadcasted in step $2(\mathrm{~b})$ or step $2(\mathrm{~d})$;

otherwise, $p_{i}$ broadcasts "accept".

3. If less than $3 e_{f}+2 e_{c}+1$ parties in $\mathcal{G}$ broadcast "accept", then the dealer is disqualified. Otherwise, note that two polynomials $g_{i}(x)$ and $h_{i}(x)$ are associated with each uncorrupted party $p_{i}$ in $\mathcal{G}$ (If the polynomials are not made public in step $2(\mathrm{e})$, then the polynomials associated with $p_{i}$ are the two polynomials $p_{i}$ received in step (1)). Each party $p_{i} \in \mathcal{G}$ sends $h_{i}(j)$ to all parties $p_{j} \notin \mathcal{G}$.

4. For each party $p_{i} \notin \mathcal{G}, p_{i}$ constructs a degree $e_{f}$ polynomial $g_{i}(x)$ by using the Reed-Solomon error-correction interpolation procedure on the values it received in last step (If $p_{i}$ cannot construct such polynomial or $p_{i}$ receives less than $2 e_{f}+e_{c}+1$ shares, then $p_{i}$ aborts).

5. Each party $p_{i}$ outputs $g_{i}(0)$.

VSS-reconstruct $\left(g_{i}(0)\right)$

1. Party $p_{i}$ sends $g_{i}(0)$ to all parties.

2. Let $S S^{i}$ be the set of secret shares $p_{i}$ receives in last round. If $\left|S S^{i}\right|<3 e_{f}+1$, then $p_{i}$ aborts else $p_{i}$ reconstructs a polynomial $h_{0}(x)$ of degree $e_{f}$ by using the Reed-Solomon error-correction interpolation on the set $S S^{i}$ and outputs $h_{0}(0)$.

We now proceed to prove that the above protocol achieves VSS.

- Privacy: Consider an uncorrupted dealer. If an uncorrupted party $p_{i}$ broadcasts "complaint: i,j" in step 2(b), then $p_{j}$ must be a corrupted party. It is easy to see that if a party $p_{i}$ broadcasts a complaint in step $2(\mathrm{~d})$, then $p_{i}$ is a corrupted party. Therefore, all the information broadcasted by an uncorrupted dealer on $f$, if any, is a subset of the shares the corrupted parties entitled to receive in step 1 . Since the secret is shared by a random bivariate polynomial of degree $e_{f}$, we conclude that the view of the adversary is independent of $s$ during the sharing phase.

- Agreement: Note that the decision of disqualifying a dealer is completely dependent on the messages broadcasted by the parties. If an uncorrupted party does not abort by the end of the sharing phase, then by the agreement property of broadcast, the values it received from the broadcasts are same as those received by fault-free parties. Hence agreement follows.

- Correctness: We first consider a fault-free dealer. All fault-free parties will be in $\mathcal{G}$. Since $n \geq 4 e_{f}+3 e_{c}+1$, it follows that $|\mathcal{G}| \geq 3 e_{f}+2 e_{c}+1$. In addition, all fault-free parties broadcast "accept" in step 2(f). For a constrained party $p_{i}$ that is not in $\mathcal{G}$, it is easy to see that $g_{i}(x)$ reconstructed in step 3 (if $p_{i}$ does not abort) is equal to $f(x, i)$.

Next we consider a constrained dealer that is not disqualified. For an uncorrupted party $p_{i} \in \mathcal{G}$ that does not abort by step $2(\mathrm{f})$, it is easy to see that $g_{i}(x)=f(x, i)$ and $h_{i}(x)=f(x, i)$. If the dealer is not disqualified, then $\geq 3 e_{f}+2 e_{c}+1$ parties broadcasts "accept" in step $2(\mathrm{f})$. Let $\mathcal{G}^{\prime}$ be the set 
of fault-free parties among these $\geq 3 e_{f}+2 e_{c}+1$ parties. $\left|\mathcal{G}^{\prime}\right| \geq 2 e_{f}+e_{c}+1$. It then follows that every fault-free party (or constrained party that does not abort in step 3) $p_{i}$ that is not in $\mathcal{G}$ can reconstruct $g_{i}(x)=f(x, i)$. An uncorrupted party $p_{i}$ (if it does not abort) will then output $f(0, i)$ in step 4 .

- Commitment: We consider the case of a non-disqualified corrupted dealer. If a corrupted dealer is not disqualified, then at least $2 e_{f}+e_{c}+1$ fault-free parties broadcast "accept" in step $2(\mathrm{f})$. Let $\mathcal{G}^{\prime}$ be the set of such fault-free parties. Following [13, Lemma 2], there exists a bivariate polynomial $f^{\prime}$ of degree $e_{f}$ in each variable such that for all $p_{i} \in \mathcal{G}^{\prime}, g_{i}(x)=f^{\prime}(x, i)$ and $h_{i}(x)=f^{\prime}(i, x)$. Now consider an uncorrupted party $p_{j} \in \mathcal{G}$ but not in $\mathcal{G}^{\prime}$. There are 2 possible scenarios:

- $p_{j}$ broadcasts a complaint in step $2(\mathrm{~d}) \cdot g_{j}(x)$ and $h_{j}(x)$ are made public in step 2(e). For all $p_{i} \in \mathcal{G}^{\prime}, g_{j}(i)=h_{i}(j)$ and $h_{j}(i)=g_{i}(j)$. Hence it follows that $g_{j}(x)=f^{\prime}(x, j)$ and $h_{i}(x)=f^{\prime}(x, i)$.

- $p_{j}$ does not broadcast a complaint in step 2(d). If $p_{j}$ does not abort in step 2(b), then $h_{j}(i)=f_{i}(j)$ and $f_{j}(i)=h_{i}(j)$ for at least $2 e_{f}+e_{c}+1-$ $\left(e_{f}+e_{c}\right)=e_{f}+1$ parties $p_{i} \in \mathcal{G}^{\prime}$. Since $f^{\prime}$ is a bivariate polynomial of degree $e_{f}$, it follows that $h_{j}(x)=f^{\prime}(j, x)$ and $g_{j}(x)=f^{\prime}(x, j)$. Therefore if $p_{j}$ does not broadcast a complaint in step 2(d), it will not broadcast "reject" in step 2(f).

We conclude that for all uncorrupted parties $p_{i} \in \mathcal{G}$ that do not abort by step $2, h_{i}(x)=f^{\prime}(i, x)$ and $g_{i}(x)=f^{\prime}(x, i)$. It is easy to see that if an uncorrupted party $p_{j} \notin \mathcal{G}$ does not abort by step $3, p_{j}$ can reconstruct $g_{j}(x)=f^{\prime}(x, j)$.

Hence it follows that an uncorrupted party $p_{i}$ (if it does not abort) outputs $f^{\prime}(0, i)$ in step 4.

It also follows that all uncorrupted parties that do not abort output $h^{\prime}(0)$ by the end of VSS-reconstruct.

\section{$5 \quad \mathrm{MPC}$}

We now construct a MPC protocol following the paradigm in [3]. On a high level, each party shares its private input, evaluates the circuit gate by gate, and then reconstructs the outputs.

Input Phase: Every party shares its private input using VSS-share. If a party is disqualified (when it plays the role of the dealer), then the party is added to the set $\mathcal{D}$. Note that by the end of the input phase, all uncorrupted parties that do not abort have the same view on $\mathcal{D}$.

Circuit Evaluation: All parties that do not abort evaluate the circuit $g^{\mathcal{D}}$ gate by gate. A party who was disqualified in the input phase does not take part in this phase and all other parties will ignore the messages sent from that party. It suffices to consider the addition and multiplication gates. The evaluation procedures are very similar to the one in $[7$, section 4.52$]$ and we omit the details here. 
Output phase: Reconstructing output is easy. For each output wire, each party sends its share to the party who is entitled to receive the output. The corresponding party then reconstructs the output from the shares it received using error correction. A party aborts if it receives less than $3 e_{f}+1$ entitled shares.

\section{Extending to the Case of $\delta<\frac{1}{6}$}

We describe how to extend the results from the previous sections (which assume $\delta=0$ ) to the case of $\delta<\frac{1}{6}$, at the expense of increasing the round complexity by a factor of 2 . More precisely, we show how to compile a protocol $\Pi$ for $\delta=0$ into a protocol $\Pi^{\prime}$ for $\delta<\frac{1}{6}$.

Our broadcast protocol $\Pi$ for $\delta=0$ assumes $n \geq 3 e_{f}+2 e_{c}$ but does not assume secure channels. If $p_{i}$ is supposed to send $p_{j}$ a message $m$ in $\Pi$, then the followings are carried out in $\Pi^{\prime}$ :

- $p_{i}$ sends $m$ to all parties who then forward the message to $p_{j}$.

- If there exists $m^{\prime}$ such that $p_{j}$ receives $\geq \frac{4}{3} e_{f}$ copies of them, then $p_{j}$ sets $m=m^{\prime}$ else $m=\phi$.

Consider the following two cases:

1. Both $p_{i}$ and $p_{j}$ are fault-free parties: since $n \geq 3 e_{f}+2 e_{c}$, at least $\left(2 e_{f}+\right.$ $\left.e_{c}\right)(1-\delta)$ fault-free parties receive $m$ from $p_{i}$. The number of copies of $m$ $p_{j}$ received is at least $\left(2 e_{f}+e_{c}\right)(1-2 \delta)$ which is greater than $\frac{4}{3} e_{f}$ if $\delta<\frac{1}{6}$. Hence $p_{j}$ can receive $m$ from $p_{i}$.

2. At least one of the $p_{i}$ and $p_{j}$ is a constrained party: suppose $p_{j}$ receives $m^{\prime}$ from $p_{i}$ in $\Pi^{\prime}$ and $m^{\prime} \neq \phi$. Since $p_{j}$ receives at least $\frac{4}{3} e_{f}$ copies of $m^{\prime}$, at least $\frac{1}{3} e_{f}$ copies are from uncorrupted parties. Hence $m^{\prime}=m$ (assuming $e_{f} \geq 3$ ).

For VSS and MPC protocols, we assume $n \geq 4 e_{f}+3 e_{c}+1$ but we also assume secure channels. If $p_{i}$ is supposed to send $p_{j}$ a message $m$ in $\Pi$, then the following steps are carried out in $\Pi^{\prime}$ :

- $p_{i}$ picks a random polynomial $h(x)$ of degree $e_{f}$ such that $h(0)=m \cdot p_{i}$ sends $h(k)$ to $p_{k}$ who then forwards the share to $p_{j}$.

- Based on the shares $p_{j}$ received, using the Reed-Solomon error-correction interpolation procedure, $p_{j}$ constructs a polynomial $h^{\prime}(x)$ of degree $e_{f}$ such that at least $2 e_{f}+1$ shares are on $h^{\prime}(x)$. If $p_{j}$ cannot construct such polynomial, then $p_{j}$ sets $m=\phi$ else $m=h^{\prime}(0)$.

First we note that if $p_{i}$ is uncorrupted, then the view of the adversary is independent of $m$ since $h$ is a random polynomial of degree $e_{f}$. Second, if both $p_{i}$ and $p_{j}$ are uncorrupted and $p_{j}$ does not set $m=\phi$, then $p_{j}$ receives $2 e_{f}+1$ shares that are on $h^{\prime}(x)$. $e_{f}+1$ of these shares are from uncorrupted parties. Hence $h^{\prime}(x)=h(x)$ since both $h^{\prime}(x)$ and $h(x)$ are of degree $e_{f}+1$. Finally, if both $p_{i}$ and $p_{j}$ are fault-free parties, then $p_{j}$ will receive at least $\left(3 e_{f}+2 e_{c}+1\right)(1-2 \delta) \geq$ $2 e_{f}+1$ (assuming $\delta<\frac{1}{6}$ and $e_{c} \geq 1$ ) correct shares. On the other hand, $p_{j}$ will receive at most $e_{f}$ corrupted shares. Hence $p_{j}$ can always reconstruct $h(x)$ using error-correction. 


\section{Conclusion and Open Problems}

In this paper, we consider a communication model where message delivery is neither always guaranteed nor always in the hands of the adversary. We has developed broadcast and VSS protocols under this model. However, we do not know if the bounds are tight. Another interesting direction is to consider what is achievable if the global clock is removed from the model.

\section{Acknowledgments}

The author thanks Omer Horvitz, Jonathan Katz, Ruggero Morselli, TsuenWan "Johnny" Ngan and Ji Sun Shin for helpful comments and encouragement. In particular, discussions on the communication model with Ruggero Morselli and Tsuen-Wan "Johnny" Ngan are very helpful. The author also thanks the anonymous referees for providing useful references and thoughtful comments.

\section{References}

1. D. Beaver. Multiparty protocols tolerating half faulty processors. In G. Brassard, editor, Advances in Cryptology - CRYPTO '89, 9th Annual International Cryptology Conference, volume 435 of Lecture Notes in Computer Science, pages 560-572. Springer, 1989.

2. M. Ben-Or, R. Canetti, and O. Goldreich. Asynchronous secure computation. In STOC '93: Proceedings of the twenty-fifth annual ACM symposium on Theory of computing, pages 52-61, New York, NY, USA, 1993. ACM Press.

3. M. Ben-Or, S. Goldwasser, and A.Wigderson. Completeness theorems for noncryptographic fault-tolerant distributed computations. In Proceedings of the 20th annual ACM symposium on Theory of computing, pages 1-10, 1988.

4. M. Ben-Or, B. Kelmer, and T. Rabin. Asynchronous secure computations with optimal resilience (extended abstract). In PODC '94: Proceedings of the thirteenth annual ACM symposium on Principles of distributed computing, pages 183-192, New York, NY, USA, 1994. ACM Press.

5. P. Berman, J. A. Garay, and K. J. Perry. Towards optimal distributed consensus (extended abstract). In Proceedings of the 30th Annual Symposium on Foundations of Computer Science, pages 410-415. IEEE, 1989.

6. M. Biely. Optimal agreement protocol in malicious faulty processors and faulty links. IEEE Transactions on Knowledge and Data Engineering, 4(3):266-280, 1992.

7. R. Canetti. Studies in Secure Multiparty Computation and Applications. PhD thesis, Weizmann Institute of Science, Rehovot 76100, Israel, June 1995.

8. R. Canetti. Universally composable security: A new paradigm for cryptographic protocols. In FOCS '01: Proceedings of the 42nd IEEE symposium on Foundations of Computer Science, page 136, Washington, DC, USA, 2001. IEEE Computer Society.

9. R. Canetti, S. Halevi, and A. Herzberg. Maintaining authenticated communication in the presence of break-ins. In PODC '9\%: Proceedings of the sixteenth annual ACM symposium on Principles of distributed computing, pages 15-24, New York, NY, USA, 1997. ACM Press. 
10. R. Canetti, Y. Lindell, R. Ostrovsky, and A. Sahai. Universally composable twoparty and multi-party secure computation. In STOC '02: Proceedings of the thiryfourth annual ACM symposium on Theory of computing, pages 494-503, New York, NY, USA, 2002. ACM Press.

11. D. Chaum, C. Crepeau, and I. Damgard. Multiparty unconditionally secure protocols. In STOC '88: Proceedings of the twentieth annual ACM symposium on Theory of computing, pages 11-19, New York, NY, USA, 1988. ACM Press.

12. R. Cramer, I. Damgård, S. Dziembowski, M. Hirt, and T. Rabin. Efficient multiparty computations secure against an adaptive adversary. In J. Stern, editor, Advances in Cryptology - EUROCRYPT '99, volume 1592 of Lecture Notes in Computer Science, pages 311-326. Springer-Verlag, May 1999.

13. P. Feldman and S. Micali. An optimal probabilistic protocol for synchronous byzantine agreement. SIAM J. Comput., 26(4):873-933, 1997.

14. J. A. Garay and K. J. Perry. A continuum of failure models for distributed computing. In Proceedings of the 6th International Workshop on Distributed Algorithms, pages 153-165. Springer-Verlag, 1992. Full version availabe at http://cm.belllabs.com/who/garay/continuum.ps.

15. R. Gennaro, M. O. Rabin, and T. Rabin. Simplified vss and fast-track multiparty computations with applications to threshold cryptography. In PODC '98: Proceedings of the seventeenth annual ACM symposium on Principles of distributed computing, pages 101-111, New York, NY, USA, 1998. ACM Press.

16. O. Goldreich, S. Micali, and A. Wigderson. How to play any mental game. In Proceedings of the nineteenth annual ACM conference on Theory of computing, pages 218-229. ACM Press, 1987.

17. S. Goldwasser and Y. Lindell. Secure computation without agreement. In DISC '02: Proceedings of the 16th International Conference on Distributed Computing, pages 17-32, London, UK, 2002. Springer-Verlag.

18. Y. T. Kalai, Y. Lindell, and M. Prabhakaran. Concurrent general composition of secure protocols in the timing model. In STOC '05: Proceedings of the thirtyseventh annual ACM symposium on Theory of computing, pages 644-653, New York, NY, USA, 2005. ACM Press.

19. A. D. Keromytis, V. Misra, and D. Rubenstein. Sos: secure overlay services. $S I G$ COMM Comput. Commun. Rev., 32(4):61-72, 2002.

20. P. R. Parvédy and M. Raynal. Optimal early stopping uniform consensus in synchronous systems with process omission failures. In Proceedings of the sixteenth annual ACM symposium on Parallelism in algorithms and architectures, pages 302 310. ACM Press, 2004.

21. K. J. Perry and S. Toueg. Distributed agreement in the presence of processor and communication faults. IEEE Trans. Softw. Eng., 12(3):477-482, 1986.

22. T. Rabin and M. Ben-Or. Verifiable secret sharing and multiparty protocols with honest majority. In STOC '89: Proceedings of the twenty-first annual ACM symposium on Theory of computing, pages 73-85, New York, NY, USA, 1989. ACM Press.

23. U. Schmid, B. Weiss, and J. Rushby. Formally verified byzantine agreement in presence of link faults. In ICDCS '02: Proceedings of the 22 nd International Conference on Distributed Computing Systems (ICDCS'02), page 608, Washington, DC, USA, 2002. IEEE Computer Society.

24. P. Thambidurai and Y.-K. Park. Interactive consistency with multiple failure modes. In Proceedings of the 7th Reliable Distributed Systems Symposium, pages 93-100, 1988. 
25. K. Q. Yan, Y. H. Chin, and S. C. Wang. Optimal agreement protocol in malicious faulty processors and faulty links. IEEE Transactions on Knowledge and Data Engineering, 4(3):266-280, 1992.

26. A. C.-C. Yao. Protocols for secure computations. In FOCS '82: Proceedings of the 23rd Symposium on Foundations of Computer Science, pages 160-164, Los Alamitos, CA, USA, 1982. IEEE Computer Society Press. 